\title{
Modelos de regressão para estimativa da área foliar de espécies brasileiras do gênero Tillandsia L. (Bromeliaceae) ${ }^{1}$
}

\author{
PATRÍCIA GABARRA MENDONÇA², JOSÉ CARLOS BARBOSA ${ }^{3}$ e MARIA ESMERALDA SOARES PAYÃO DEMATTÊ4,5
}

\begin{abstract}
RESUMO
O objetivo deste trabalho foi ajustar equações de regressão para estimativa da área foliar de Tillandsia spp. nativas do Brasil. Determinaram-se o comprimento ao longo da nervura principal (C), a largura máxima (L) e a área foliar real de, no mínimo, 100 folhas de cada espécie, mantidas intactas nas plantas. Calcularam-se relações lineares existentes entre a área foliar real e as dimensões C, L e CL (produto entre o comprimento e a largura da folha). O modelo de regressão linear com intercepto foi o mais adequado, e as equações recomendadas, sendo $\mathrm{AFE}=$ área foliar estimada, foram: para Tillandsia carminea, $\mathrm{AFE}=$ $0,281188+0,562219 \mathrm{CL}\left(\mathrm{R}^{2}=0,7296\right) ;$ Tillandsia dura, $\mathrm{AFE}=0,891231+0,412457 \mathrm{CL}\left(\mathrm{R}^{2}=0,8432\right)$; Tillandsia gardneri, $\mathrm{AFE}=2,54412+0,445525$ $\mathrm{CL}\left(\mathrm{R}^{2}=0,8662\right)$; Tillandsia geminiflora, $\mathrm{AFE}=$ $0,674484+0,444418 \mathrm{CL}\left(\mathrm{R}^{2}=0,8474\right) ;$ Tillandsia globosa, $\mathrm{AFE}=1,9494+0,3804 \mathrm{CL}\left(\mathrm{R}^{2}=0,8178\right)$; Tillandsia grazielae, $\mathrm{AFE}=0,518512+0,530259 \mathrm{CL}$ $\left(\mathrm{R}^{2}=0,7608\right)$; Tillandsia kautskyi, $\mathrm{AFE}=0,59338+$ $0,374353 \mathrm{CL}\left(\mathrm{R}^{2}=0,6931\right)$; Tillandsia reclinata, $\mathrm{AFE}=$ $0,0379111+0,641623 \mathrm{CL}\left(\mathrm{R}^{2}=0,9169\right) ;$ Tillandsia stricta, $\mathrm{AFE}=0,3937+0,4277 \mathrm{C}\left(\mathrm{R}^{2}=0,8661\right)$, ou $\mathrm{AFE}=1,21336+0,385727 \mathrm{CL}\left(\mathrm{R}^{2}=0,8607\right)$; Tillandsia tenuifolia, $\mathrm{AFE}=0,991226+0,402221 \mathrm{CL}\left(\mathrm{R}^{2}=\right.$ 0,7523).
\end{abstract}

Palavras-chave: bromélia, Bromeliaceae, modelos de predição.

\section{ABSTRACT \\ Regression models for leaf area estimation of Brazilian species of the genus Tillandsia $L$. (Bromeliaceae)}

The objective of this study was to calculate adequate regression equations for estimation of leaf area of Tillandsia spp. native from Brazil. Leaf length along the central vein (C), maximum leaf width (L) and real leaf area were measured from at least 100 leaves for each species. Leaves were maintained intact at the plants. Relationships between the real leaf area and leaf dimensions $\mathrm{C}, \mathrm{L}$ and $\mathrm{CL}$ (leaf length $\mathrm{x}$ leaf maximum width) were calculated with regression models. The linear regression model with intercept was the most adequate, and the recommended equations for each species, being LA = estimated leaf area, were: Tillandsia carminea, $\mathrm{LA}=0,281188+0,562219 \mathrm{CL}\left(\mathrm{R}^{2}=0,7296\right)$; Tillandsia dura, $\mathrm{LA}=0,891231+0,412457 \mathrm{CL}\left(\mathrm{R}^{2}=\right.$ $0,8432)$; Tillandsia gardneri, $\mathrm{LA}=2,54412+0,445525$ $\mathrm{CL}\left(\mathrm{R}^{2}=0,8662\right)$; Tillandsia geminiflora, $\mathrm{LA}=0,674484$ $+0,444418 \mathrm{CL}\left(\mathrm{R}^{2}=0,8474\right)$; Tillandsia globosa, $\mathrm{LA}=$ $1,9494+0,3804 \mathrm{CL}\left(\mathrm{R}^{2}=0,8178\right)$; Tillandsia grazielae, $\mathrm{LA}=0,518512+0,530259 \mathrm{CL}\left(\mathrm{R}^{2}=0,7608\right)$; Tillandsia kautskyi, $\mathrm{LA}=0,59338+0,374353 \mathrm{CL}\left(\mathrm{R}^{2}=0,6931\right)$; Tillandsia reclinata, $\mathrm{LA}=0,0379111+0,641623 \mathrm{CL}$ $\left(\mathrm{R}^{2}=0,9169\right) ;$ Tillandsia stricta, $\mathrm{LA}=0,3937+0,4277$ $\mathrm{C}\left(\mathrm{R}^{2}=0,8661\right)$, or $\mathrm{LA}=1,21336+0,385727 \mathrm{CL}\left(\mathrm{R}^{2}=\right.$ $0,8607)$; Tillandsia tenuifolia, $\mathrm{LA}=0,991226+0,402221$ $\mathrm{CL}\left(\mathrm{R}^{2}=0,7523\right)$.

Key words: bromeliads, Bromeliaceae, prediction models.

\footnotetext{
${ }^{1}$ Extraído da Dissertação de Mestrado da primeira autora.

2 Departamento de Biologia Aplicada à Agropecuária, Faculdade de Ciências Agrárias e Veterinárias, Unesp, Campus de Jaboticabal. Via de Acesso Prof. Paulo Donato Castellane, s/n, 14884-900, Jaboticabal (SP).

${ }^{3}$ Departamento de Ciências Exatas, Faculdade de Ciências Agrárias e Veterinárias, Unesp, Campus de Jaboticabal. Via de Acesso Prof. Paulo Donato Castellane, $\mathrm{s} / \mathrm{n}, 14884-900$, Jaboticabal (SP).

${ }^{4}$ Departamento de Produção Vegetal, Faculdade de Ciências Agrárias e Veterinárias, Unesp, Campus de Jaboticabal. Via de Acesso Prof. Paulo Donato Castellane, $\mathrm{s} / \mathrm{n}, 14884-900$, Jaboticabal (SP).

${ }^{5}$ Bolsista do CNPq. Autora para correspondência.
} 


\section{INTRODUÇÃO}

A área foliar relacionada com a capacidade fotossintetizante e com o acúmulo de matéria seca é utilizada, principalmente, em análise de crescimento e em estudos de fenologia e nutrição de plantas.

Para determinação da área foliar, diversos métodos podem ser utilizados (BENINCASA, 1988). Dentre eles, os não destrutivos permitem efetuar avaliações durante todo o ciclo de vida da planta e são de interesse em estudos nos quais o número de plantas disponíveis é limitado. Um dos métodos não destrutivos para estimar a área foliar é relacioná-la com medidas lineares das folhas por meio de equações de regressão. Esse método, simples, rápido e de baixo custo, é ainda mais interessante quando a espécie vegetal apresenta folhas como as das bromélias, suculentas e côncavas, que tornam impraticável o uso de aparelhos como o integrador de área foliar.

A área foliar de Bromeliaceae tem sido pouco estudada. Para o gênero Tillandsia L., apenas um trabalho foi encontrado na literatura revista, desenvolvido por BARBOSA et al. (1995) para T. stricta Sol. var. stricta. Esses autores demonstraram que é possível estimar a área foliar da espécie por meio de equações de regressão com base em dimensões da folha.

O gênero Tillandsia é, dentro da família Bromeliaceae, o que tem maior número de espécies, cerca de 402, distribuídas em toda a região neotropical (NUNES, 1997). As espécies de Tillandsia são plantas caulescentes ou acaulescentes, epífitas ou rupícolas, de hábito muito variado. Características botânicas do gênero são citadas por SMITH \& DOWNS (1977), LEME \& MARIGO (1993), BENZING (1994), NUNES (1997) e TILL (2000).

O principal interesse dessas espécies é ornamental. CATHCART (1994) citou algumas com potencial para exportação, entre elas Tillandsia stricta, T. tenuifolia, T. gardneri e T. geminiflora, todas brasileiras. No mercado interno, também é possível ampliar a comercialização de tilândsias, porque são bromélias que não formam "tanque" na parte central da planta e, por não acumularem água, não há motivo para associá-las à proliferação do mosquito Aedes aegypti, transmissor de dengue.

Entre as tilândsias brasileiras, T. kautskyi, T. carminea, $T$. grazielae e $T$. reclinata estão ameaçadas de extinção (CITES, 2001; BDT, 2001; IBAMA, 2001; IUCN, 2001). Essas espécies ainda não são cultivadas em escala comercial no Brasil (ANDRADE \& DEMATTÊ, 1999). Embora os esforços para conser- vação in situ sejam indispensáveis, o cultivo é uma das práticas que podem contribuir para a conservação de espécies ameaçadas. Para desenvolver métodos de cultivo adequados, há necessidade de conhecer melhor o comportamento biológico da espécie, e a área foliar é um parâmetro de importância fundamental nesses estudos.

Partindo da hipótese de que é possível estimar com precisão satisfatória a área foliar de diversas espécies do gênero Tillandsia por método não destrutivo, a exemplo do que já foi comprovado por BARBOSA et al. (1995) para T. stricta var. stricta, o objetivo do presente trabalho foi determinar equações de regressão para estimativa da área foliar de espécies de Tillandsia brasileiras, por meio das relações existentes entre a área foliar real e variáveis dimensionais (comprimento e maior largura das folhas).

\section{MATERIAL E MÉTODOS}

As espécies estudadas, todas brasileiras, pertencem ao gênero Tillandsia e são as seguintes: Tillandsia carminea W. Till, Tillandsia dura Baker, Tillandsia gardneri Lindl., Tillandsia geminiflora Brongn., Tillandsia globosa Wawra, Tillandsia grazielae Sucre \& Braga, Tillandsia kautskyi E. Pereira, Tillandsia reclinata E. Pereira \& Martinelli, Tillandsia stricta Sol. ex Sims e Tillandsia tenuifolia L.

As plantas utilizadas haviam sido coletadas em áreas silvestres próximas a Petrópolis, $\mathrm{RJ}$; foram apreendidas e estavam sob a guarda do Orquidário Binot, Petrópolis, RJ, que as doou, com autorização legal, à Faculdade de Ciências Agrárias e Veterinárias da Universidade Estadual Paulista (Unesp), Campus de Jaboticabal, SP, onde estão em cultivo no Viveiro Experimental de Plantas Ornamentais e Florestais, em telado com $70 \%$ de interceptação da luz solar. Jaboticabal é local de ocorrência de bromélias (PINTO et al., 1994 e 1995) e de condições favoráveis ao seu desenvolvimento. De acordo com os registros da Estação Agroclimatológica do Departamento de Ciências Exatas da FCAV-Unesp (FCAV-UNESP, 2004), em Jaboticabal, no período de 1971 a 2000, os meses mais quentes foram janeiro e fevereiro, com temperaturas médias de $24,3^{\circ} \mathrm{C}$, e o mês mais frio foi junho, com temperatura média de $18,6^{\circ} \mathrm{C}$; os meses com maior e menor umidade relativa do ar foram, respectivamente, janeiro (média de 79,0\%) e agosto (média de 58,2\%); o total médio anual de chuvas foi de 1424,6 mm, e a insolação (número de horas de brilho solar) média anual, de $2585,8 \mathrm{~h}$. 
Informações botânicas sobre as espécies estudadas são encontradas em SMITH \& DOWNS (1955 e 1977), PEREIRA (1974), SUCRE BENJAMIN \& BRAGA (1975), PEREIRA \& MARTINELLI (1982), REITZ (1983) e EHLERS (1996). Tillandsia dura, T. geminiflora, T. globosa, T. kautskyi e T. tenuifolia são epífitas; T. carminea, T. grazielae e $T$. reclinata, rupícolas; $T$. gardneri e T. tenuifolia, epífitas e rupícolas facultativas; T. stricta é epífita e rupícola ocasional (GRAEFF; TILL; GOUDA ${ }^{6}$ )

Para medir as dimensões foliares e determinar a área foliar real, foram desenhadas folhas plenamente desenvolvidas, escolhidas ao acaso. O número de folhas medidas para cada espécie, que dependeu da quantidade de plantas disponíveis, foi: T. carminea, 183; T. dura, 100; T. gardneri, 200; T. geminiflora, 201; T. globosa, 202; T. grazielae, 151; T. kautskyi, 212; T. reclinata, 102; T. stricta, 205; T. tenuifolia, 207. Os desenhos foram feitos em filme de plástico transparente colocado sobre a folha, traçando-se seu contorno com caneta especial para escrever em transparências para retroprojetor. Posteriormente, foram transferidos para papel.

Foram medidos, para cada folha desenhada, o comprimento ao longo da nervura principal excluindo a bainha foliar $(\mathrm{C})$ e a largura máxima perpendicular à nervura principal (L). A área foliar real foi determinada pela medição da área desenhada no papel, utilizandose sistema de análise digital de imagem (Digital Image Analysis System Version 1.12 - Delta - T Devices). Em seguida, foram determinadas, com o auxílio de modelos de regressão, as equações ideais para representar a área foliar real em função das dimensões lineares $(\mathrm{x}=\mathrm{C}$ e $\mathrm{x}=\mathrm{L})$ e do produto entre comprimento e largura $(x=C L)$ da folha de cada espécie estudada. Estabeleceu-se como critério que, se a regressão linear fosse significativa, esse seria o modelo escolhido.

\section{RESULTADOS E DISCUSSÃO}

Os gráficos de regressão linear entre CL (produto entre o comprimento e a largura máxima da folha) e a área foliar real de Tillandsia spp., apresentados na Figura 1, mostram que o modelo de regressão linear simples $(\mathrm{Y}=\mathrm{a}+\mathrm{bX})$ apresentou boa aproximação da área foliar estimada com a área foliar real de todas as espécies estudadas. Por sua simplicidade, esse modelo foi escolhido para o cálculo das equações de estimativa da área foliar, apresentadas na Tabela 1 .

Das equações ajustadas para $T$. stricta, a que apresentou maior Coeficiente de Determinação foi a que relacionou área foliar real somente com o comprimento da folha $\left(\mathrm{R}^{2}=0,8661\right.$, Tabela 1$)$. Esse resultado é interessante, pois indica que basta uma medida na folha para estimar sua área. Além da economia de tempo, a possibilidade de dano à folha diminui.

Nos cálculos para as demais espécies, quando se considerou apenas o comprimento $(\mathrm{C})$ ou a maior largura (L), as equações obtidas não apresentaram boa aproximação para a estimativa da área foliar real, isto é, os Coeficientes de Determinação $\left(\mathrm{R}^{2}\right)$ foram baixos.

Tabela 1. Equações de regressão para estimativa da área foliar de Tillandsia spp. e respectivos Coeficientes de Determinação $\left(\mathrm{R}^{2}\right)$, valores obtidos no Teste F para o modelo ajustado e Quadrados Médios do Resíduo (QMR) da análise de variância da regressão

\begin{tabular}{|c|c|c|c|c|}
\hline Espécie & Equação de regressão ${ }^{1}$ & $\mathrm{R}^{2}$ & Teste F & QMR \\
\hline Tillandsia carminea & $\mathrm{AFE}=0,281188+0,562219 \mathrm{CL}$ & 0,7296 & $488,4425 * 2$ & 0,043850 \\
\hline Tillandsia dura & $\mathrm{AFE}=0,891231+0,412457 \mathrm{CL}$ & 0,8432 & $527,1084 * *$ & 0,270260 \\
\hline Tillandsia gardneri & $\mathrm{AFE}=2,54412+0,445525 \mathrm{CL}$ & 0,8662 & $1281,8570 * *$ & 2,992590 \\
\hline Tillandsia geminiflora & $\mathrm{AFE}=0,674484+0,444418 \mathrm{CL}$ & 0,8474 & $1105,4810^{* *}$ & 0,268930 \\
\hline Tillandsia globosa & $\mathrm{AFE}=1,9494+0,3804 \mathrm{CL}$ & 0,8178 & $976,6900 * *$ & 0,466300 \\
\hline Tillandsia grazielae & $\mathrm{AFE}=0,518512+0,530259 \mathrm{CL}$ & 0,7608 & $473,9436 * *$ & 0,340020 \\
\hline Tillandsia kautskyi & $\mathrm{AFE}=0,59338+0,374353 \mathrm{CL}$ & 0,6931 & $474,3668 * *$ & 0,255620 \\
\hline Tillandsia reclinata & $\mathrm{AFE}=0,0379111+0,641623 \mathrm{CL}$ & 0,9169 & $1102,8260 * *$ & 0,014215 \\
\hline \multirow[t]{2}{*}{ Tillandsia stricta } & $\mathrm{AFE}=1,21336+0,385727 \mathrm{CL}$ & 0,8607 & $1254,5650^{* *}$ & 0,492060 \\
\hline & $\mathrm{AFE}=0,3937+0,4277 \mathrm{C}$ & 0,8661 & $1320,4400 * *$ & 0,470800 \\
\hline Tillandsia tenuifolia & $\mathrm{AFE}=0,991226+0,402221 \mathrm{CL}$ & 0,7523 & $622,6440 * *$ & 0,167640 \\
\hline
\end{tabular}

${ }^{1} \mathrm{AFE}=$ área foliar estimada; $\mathrm{C}=$ comprimento da folha ao longo da nervura principal; $\mathrm{L}=$ largura máxima perpendicular à nervura principal.

$2 * *=$ significativo a $1 \%$ de probabilidade.

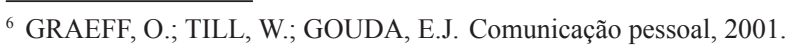



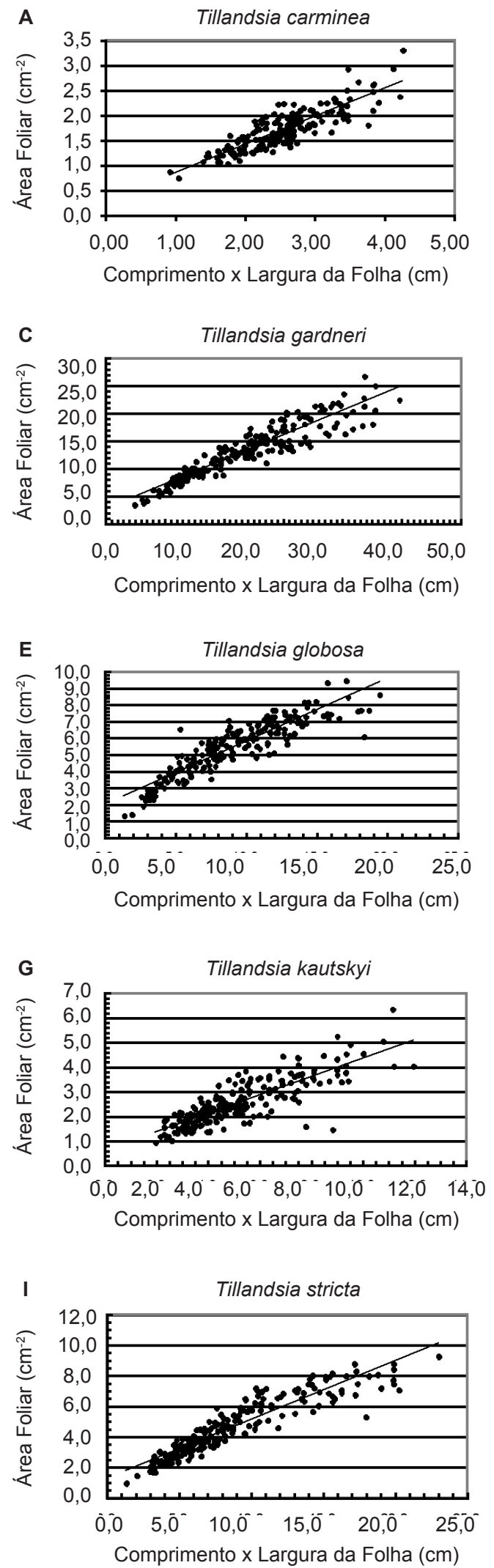
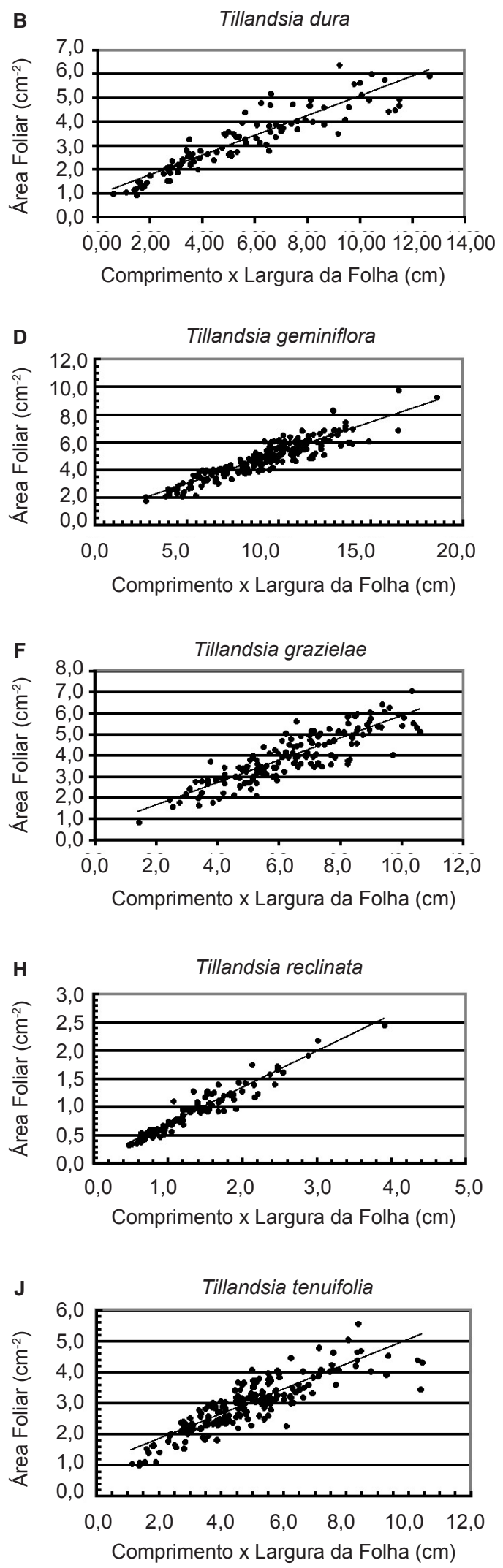

Figura 1. Gráficos de regressão linear entre CL (produto entre o comprimento e a largura máxima da folha) e a área

foliar real de Tillandsia spp. 
Estudos sobre dimensões foliares de espécies vegetais, especialmente de Bromeliaceae, devem levar em conta o ambiente em que a planta se desenvolveu. Existe forte relação entre as características das folhas de bromélias e a luz incidente em seu micro-habitat (COGLIATTI-CARVALHO \& ROCHA, 1999). Assim, uma mesma espécie pode apresentar folhas com características diferentes conforme cresça ao sol ou em ambiente sombreado.

Entretanto, em estudo desenvolvido por BARBOSA et al. (1995), os parâmetros da equação de regressão linear simples, recomendada para estimar a área foliar de Tillandsia stricta var. stricta (AFE $=1,00896$ $+0,37127 \mathrm{CL}\left(\mathrm{R}^{2}=0,9627\right)$, sendo $\mathrm{AFE}=$ área foliar estimada), apresentaram valores aproximados dos da equação obtida no presente trabalho. Aqueles autores também encontraram significância para a equação linear em que $\mathrm{X}$ correspondia ao comprimento da folha, assim como neste trabalho. Tillandsia stricta distribuise amplamente pela América tropical e subtropical, e apresenta grande variação de formas. O genótipo estudado por BARBOSA et al. (1995) ocorre em um fragmento florestal remanescente no município de Jaboticabal, SP, e o do presente estudo, em Petrópolis, RJ. Essa semelhança de resultados leva a supor que, para as medidas foliares consideradas, as variações dentro de uma mesma espécie, decorrentes de diferenças de ambiente, são menores do que as variações entre espécies.

\section{CONCLUSÃO}

A área foliar de Tillandsia carminea, T. dura, T. gardneri, T. geminiflora, T. globosa, T. grazielae, T. kautskyi, T. reclinata e T. tenuifolia pode ser estimada com base no produto entre comprimento e maior largura da folha, por meio de equações de regressão linear. Para estimar a área foliar de T. stricta por regressão, basta relacioná-la com o comprimento da folha.

\section{AGRADECIMENTOS}

Os autores agradecem ao Orquidário Binot, Petrópolis, RJ, pela doação das plantas utilizadas neste estudo; à Dra. Maria das Graças Lapa Wanderley, do Instituto de Botânica do Estado de São Paulo, pela identificação das espécies estudadas; ao Eng. Agr. Orlando Graeff, da Sociedade Brasileira de Bromélias, pela ajuda decisiva na obtenção das plantas; à Prof. Dra. Teresinha de Jesus Deléo Rodrigues, a Sônia Maria Raymundo Carregari e a Adão Marin, da Faculdade de Ciências Agrárias e Veterinárias, Campus de Jaboticabal, Unesp, por orientação quanto a procedimentos; à CAPES, por bolsa de pós-graduação concedida à primeira autora; ao $\mathrm{CNPq}$, por bolsa de produtividade em pesquisa concedida à terceira autora..

\section{LITERATURA CITADA}

ANDRADE, F.S.A. \& DEMATTÊ, M.E.S.P. Estudo sobre produção e comercialização de bromélias nas regiões Sul e Sudeste do Brasil. Revista Brasileira de Horticultura Ornamental, Campinas, v.5, p.97-110.

BDT. Base de dados tropical, banco de espécies. Disponível em: < http://www.bdt.org.br >, 2001. Acesso em: 27/ Ago/01.

BARBOSA, J.C.; PINTO, A.C.R.; DEMATTÊ, M.E.S.P. \& PAVANI, M.C.M.D. Equações de regressão para estimativa da área foliar de Tillandsia stricta Solander var. stricta, Bromeliaceae epífita. Científica, São Paulo, v..23, p.301-307, 1995.

BENINCASA, M.M. P. Análise de crescimento de plantas (noções básicas). Jaboticabal: FUNEP, 1988. 41p.

BENZING, D.H. How much is known about Bromeliaceae in 1994? Selbyana, Sarasota, v.15, p.1-7, 1994.

CATHCART, D. Cartas: 4 de Agosto para Carvalho. Bromélia, Rio de Janeiro, v.1, p.27-30, 1994.

CITES. Convention on International Trade in Endangered Species of Wild Fauna and Flora. Disponível em: < http://www.cites.org/eng/disc/what_is.shtml>, 2001. Acesso em: 28/Ago/01.

COGLIATTI-CARVALHO, L.C. \& ROCHA, C.F.D. da. Forma da bromélia depende da luz. Ciência Hoje, Rio de Janeiro, v.26, p.72-74, 1999.

EHLERS, R. Die bromelie. Frankfurt: Deutsche BromelienGesellschaft, 1996. 66p.

FCAV-UNESP. Resenha meteorológica do período 19712000. Disponível em: <http://www.fcav.unesp.br/ Departamentos/exatas/estacao/resenha.htm>. Aceso em: 15/5/2004.

IBAMA. Conservação e manejo sustentável da flora nativa do Brasil. da biodiversidade: lista oficial de flora ameaçada de extinção. Disponível em: <http://www2.ibama.gov.br/ flora/index0.htm>, 2001. Acesso em: 27/Ago/01. 
IUCN. Red list of threatened plants. Disponível em: $<$ http://www.unep.wcmc.org/index.html?http:// www.unep.wcmc.org/CITES/redirect.htm $\sim$ main $>, 2001$. Acesso em: 27/Ago/01.

LEME, E.M.C. \& MARIGO, L.C. Bromélias na natureza. Rio de Janeiro: Marigo Comunicação Visual, 1993. 183p.

NUNES, J.V.C. Estudo florístico e fenomorfológico de Tillandsioideae - Bromeliaceae na Serra do Cipó, M.G. São Paulo: Instituto de Biociências, Universidade de São Paulo, 1997, 129p. Dissertação (Mestrado em Ciências Biológicas)

.PEREIRA, E. Species novae in Brasilia Bromeliacearum - VII. Bradea, Rio de Janeiro, v.1, p.437-439, 1974.

PEREIRA, E. \& MARTINELLI, G. Species novae in Brasilia Bromeliacearum - XIX. Bradea, Rio de Janeiro, v.3, p.251-254, 1982.

PINTO, A.C.R.; DEMATTÊ, M.E.S.P. \& PAVANI, M.C. M.D. Levantamento de espécies arbóreas hospedeiras de epífitas pertencentes à Divisão Magnoliophyta, em fragmento de floresta no município de Jaboticabal, SP, Brasil. Ecossistema, Espírito Santo do Pinhal, v.19, p.136-148, 1994.
PINTO, A.C.R.; DEMATTÊ, M.E.S.P. \& PAVANI, M.C.M. D. Composição florística de epífitas (Magnoliophyta) em fragmento de floresta do município de Jaboticabal, SP, Brasil. Científica, São Paulo, v.23, p.283-289, 1995.

REITZ, R. Bromeliáceas e a malária-bromélia endêmica. Itajaí: Herbário Barbosa Rodrigues, 1983. 808p. (Flora Ilustrada Catarinense, 11).

SMITH, L.B. \& DOWNS, R.J. The Bromeliaceae of Brazil. Smithsonian Miscellaneous Collections, Washington, v.126, p.77-95, 1955.

SMITH, L.B. \& DOWNS, R.J. Flora Neotropica: Tillandsioideae (Bromeliaceae). New York: The New York Botanical Garden, 1977. Part 2, p.661-1492. (Monograph, 14).

SUCRE BENJAMIN, D. \& BRAGA, R. Tillandsia grazielae (Bromeliaceae) - espécie nova do Estado do Rio de Janeiro. Boletim do Museu Botânico Municipal, Curitiba, v.22, p.1-3, 1975.

TILL, W. Tillandsioideae. In: BENZING, D.H. (Ed.). Bromeliaceae: profile of an adaptative radiation. Cambridge: Cambrigde University Press, 2000. p.555-621. 